\title{
Clinical Implications of Residual Urine in Korean Benign Prostatic Hyperplasia (BPH) Patients: A Prognostic Factor for BPH-Related Clinical Events
}

\author{
Young Hwii Ko, Ji Yun Chae, Seung Min Jeong, Jae Il Kang, Hong Jae Ahn, Hyung Woo Kim', Sung Gu Kang, Hoon Ah Jang, \\ Jun Cheon, Je Jong Kim, Jeong Gu Lee \\ Department of Urology, Korea University School of Medicine, Seoul; \\ ${ }^{1}$ Department of Urology, Sahmyook Medical Center, Seoul, Korea
}

\begin{abstract}
Purpose: Although post-void residual urine (PVR) is frequently utilized clinically in patients with benign prostatic hyperplasia (BPH), mainly because of its procedural simplicity, its role as a clinical prognostic factor, predictive of treatment goals, is still under much dispute. We investigated the predictive value of PVR for BPH-related clinical events including surgery, acute urinary retention (AUR), and admission following urinary tract infection (UTI).

Methods: From January to June of 2006, patients over 50 years of age who were diagnosed with BPH for the first time at the outpatient clinic and were then treated for at least 3 years with medications were enrolled in this study. The variables of patients who underwent surgical intervention for BPH, had occurrences of AUR, or required admission due to UTI (Group 1, n=43) were compared with those of patients who were maintained with medications only (Group 2, n=266).

Results: Group 1 had a significantly higher PVR, more severe symptoms, and a larger prostate at the time of the initial diagnosis in both the univariate and the multivariate analysis. In the 39 patients who underwent BPH-related surgery, although there was a significant change in Qmax at the time of surgery (mean, 13.1 months), PVR and the symptom score remained unchanged compared with the initial evaluation. In the receiver-operating characteristic curve analysis, the area under the curve of Group 1 was in the order of prostate volume (0.834), PVR (0.712), and symptom score (0.621). When redivided by arbitrarily selected PVR cutoffs of $50 \mathrm{~mL}, 100 \mathrm{~mL}$, and $150 \mathrm{~mL}$, the relative risk of clinical BPH progression was measured as 3.93, 2.61, and 2.11. Conclusions: These data indicate that, in the symptomatic Korean population, increased PVR at baseline is a significant indicator of BPH-related clinical events along with increased symptom score or prostate volume.
\end{abstract}

Keywords: Benign prostatic hyperplasia; Disease progression; Residual urine

\section{INTRODUCTION}

Benign prostatic hyperplasia (BPH) is a progressive disease that is characterized mainly by a deterioration of symptoms over time [1] but also by the occurrence in some patients of serious outcomes, such as acute urinary retention (AUR), urinary tract infection (UTI), and the need for BPH-related surgery [2]. Goals of therapy are not only to improve symptoms and restore an acceptable quality of life but also to identify patients at risk of dis- ease progression in order to optimize their management.

Although urodynamic studies are the most sensitive tools for determination of the presence and grade of bladder outlet obstruction (BOO), these studies require invasive and uncomfortable procedures as well as the use of expensive equipment. In a clinical setting, measurement of the volume of residual urine in the bladder after voiding is a common procedure in the urological examination of patients presenting with lower urinary tract symptoms (LUTS) and has traditionally been thought to have a
Corresponding author: Jeong Gu Lee

Department of Urology, Korea University Anam Hospital, Korea University School of Medicine, Anamdong 5-ga, Seongbuk-gu, Seoul 136-705, Korea Tel: +82-2-920-5367 / Fax: +82-2-928-7864 / E-mail: jeongkl@kumc.or.kr Submitted: November 22, 2010 / Accepted after revision: December 10, 2010
This is an Open Access article distributed under the terms of the Creative Commons Attribution Non-Commercial License (http://creativecommons.org/licenses/by-nc/3.0/) which permits unrestricted non-commercial use, distribution, and reproduction in any medium, provided the original work is properly cited. 
prominent role in identifying those with an obstruction [1]. However, there is no agreement at present as to the usefulness of postvoid residual urine (PVR) as a clinical prognostic factor or in the prediction of treatment goals. Recent data from Western, longitudinal, population-based studies and placebo-controlled studies have suggested PVR as an important predictor for BPH-related surgery and the development of AUR [3-5]. However, considering that race and geographic factors influence the growth of the normal human prostate and the risk of symptomatic BPH [6], the clinical manifestation of PVR in BPH patients may also be affected by ethnicity. To the best of our knowledge, intensive epidemiological research on this issue among members of an Asian population, who generally have smaller prostates than do members of Western populations [7,8], has not yet been reported. Hence, the generalization of results from Western to Asian populations is uncertain. Therefore, in order to obtain a better understanding of this issue, we investigated the clinical implications of PVR in the Korean population by evaluating its relationship with other variables and its predictive value for BPH-related clinical events, including surgery, AUR, and admission following UTI.

\section{MATERIALS AND METHODS}

\section{Patient Selection and Data Collection}

From January to June of 2006, men over 50 years of age who had visited the outpatient clinic of a single institute for the first time with a chief complaint of LUTS, who were diagnosed as having $\mathrm{BPH}$ after routine initial evaluation, and who were then followed up for at least 3 years with medication were enrolled in this study. Routine evaluation for LUTS patients in our institute includes uroflowmetry with measurement of PVR, completion of the International Prostate Symptom Score (IPSS) questionnaire, measurement of prostate volume by transrectal ultrasonography (TRUS) after digital rectal examination, urinalysis, and measurement of serum prostate-specific antigen and creatinine.

Uroflowmetry was performed with the patient in the standing position, and the voided volume and maximal flow rate (Qmax) were recorded continuously during micturition. Only flows for which the sum of the voided volumes and PVR was at least 150 $\mathrm{mL}$ without possible artifacts were included. If the sum was below $150 \mathrm{~mL}$ at the initial evaluation, uroflowmetry was rechecked at the next visit. With the patient in a supine position, PVR was measured immediately after uroflowmetry by ultrasonography with a $7.5 \mathrm{MHz}$ linear probe. With the probe parallel two fin- gers above the symphysis pubis of the patient, an image of the largest circumference of the bladder was captured on the screen. PVR was calculated by application of the following formula: sagittal height X sagittal depth X transverse width X 0.625 [9]. After approval from the local institutional review board, these variables were surveyed from a retrospective review of medical records, and 309 patients with IPSS over 7 and Qmax below 15 $\mathrm{mL} / \mathrm{sec}$ were finally selected. We included the patients who are enough to be diagnosed as BPH by clinical symptoms and the evaluation test though the prostate volume of the selected patients are smaller than 20-30 g which is considered as normal volume in general. Patients with hematuria or pyuria, prostatespecific antigen (PSA) over $4 \mathrm{ng} / \mathrm{dL}$ at the initial screening, or prior prostate surgery or medication for LUTS were excluded in this series. In addition, this study excluded non-genitourinary conditions that affect volume of PVR such as diabetes mellitus or neurological disease.

By analysis of their medical records, the patients were divided into two groups: patients who underwent surgical interventions for $\mathrm{BPH}$, including transurethral resection of the prostate (TURP) or laser-based transurethral prostate surgery; experienced the occurrence of AUR; or required admission due to UTI during follow-up (Group 1) and counterpart patients who were maintained with medications only without occurrence of these events (Group 2). Parameters of both groups were compared and analyzed. For patients who underwent BPH-related surgery, uroflowmetry and IPSS were routinely rechecked at the time of surgery, and the change from the baseline evaluation was also analyzed.

\section{Statistical Analysis}

Statistical analysis was carried out by using a commercially available data analysis program, the SPSS ver. 12.0 (SPSS Inc., Chicago, IL, USA). Correlations between baseline PVR and other clinical parameters were determined by using the Pearson correlation test. Descriptive statistics are presented as mean \pm standard error or numbers, according to the variables. Clinical characteristics were individually entered into the univariate model and were entered into the multivariate logistic regression analyses as dependent variables. Odds ratios and P-values for trends by univariate and multivariate analysis were estimated for determination of factors that predicted $\mathrm{BPH}$-related clinical events. If a variable showed statistical significance in the multivariable analysis, we used receiver operating characteristic (ROC) curve analysis in an attempt to summarize the overall 
discriminative or diagnostic value of each variable, and the area under the ROC curve was measured. All statistical tests were two-sided, with a significant threshold set at $\mathrm{P}<0.05$.

\section{RESULTS}

The mean age of the enrolled patients was $67.5 \pm 7.8$ years; their mean PVR was $49.1 \pm 63.1 \mathrm{~mL}$, their Qmax was $9.1 \pm 4.2 \mathrm{~mL} /$ sec, their IPSS score was $18.2 \pm 7.2$, their prostate volume was $29.5 \pm 18.2 \mathrm{~g}$, and their PSA level was $1.3 \pm 0.9 \mathrm{ng} / \mathrm{dL}$. At the baseline evaluation, the amount of PVR was statistically related in a positive fashion with IPSS score (Pearson correlation coefficient, $+0.20 ; \mathrm{P}<0.001)$, prostate volume $(+0.16, \mathrm{P}=0.005)$, and serum PSA level $(+0.13, \mathrm{P}=0.022)$ and was statistically related in a negative fashion to $\mathrm{Qmax}(-0.23, \mathrm{P}<0.001)$.

During a mean follow-up of $39.8 \pm 7.2$ months, 43 patients were designated as Group 1, of whom 39 patients had undergone BPH-related surgery, 2 patients experienced AUR, and 1 patient experienced acute prostatitis. Among the 39 patients who required BPH-related surgeries, TURP was conducted in 21 patients, and laser-based surgery was performed in 18 patients. The mean duration from initial visit to surgery was $13.1 \pm 18.7$ months for BPH-related surgery patients, $30 \pm 20.4$ months for AUR patients, and 25 months for the prostatitis patient.

The characteristics of each group are summarized in Table 1. When we divided the patients into the two groups, Group 1 was significantly older and had a higher PVR, decreased Qmax, more severe symptoms, larger prostate, and higher serum PSA at the time of initial diagnosis. Multivariate analysis reconfirmed the significant differences in terms of PVR, IPSS, and prostate volume; however, the odds ratio of each variable was low (1.005, 1.07 , and 1.05, respectively). In the 39 patients who underwent BPH-related surgery, although the change in Qmax at the time of surgery was significant (from $8.3 \pm 3.3$ to $10.3 \pm 3.4 \mathrm{~mL} / \mathrm{sec}$ ), IPSS and PVR remained unchanged compared with the initial evaluation (Table 2).

For the ROC curve, the area under the curve was in the order of prostate volume (0.834), PVR (0.712), and symptom score (0.621) (Fig. 1). Using PVR, the cutoff point identifying Group

Table 2. Changes in variables in the BPH-related surgery group

\begin{tabular}{lccc}
\hline Parameter & $\begin{array}{c}\text { At baseline } \\
\text { evaluation }(\mathrm{n}=39)\end{array}$ & $\begin{array}{c}\text { At time of } \\
\text { operation }(\mathrm{n}=39)\end{array}$ & P-value \\
\hline $\mathrm{VV}(\mathrm{mL})$ & $200.6 \pm 131$ & $219.7 \pm 97$ & 0.50 \\
$\mathrm{PVR}(\mathrm{mL})$ & $69.0 \pm 57.5$ & $61.2 \pm 51.7$ & 0.56 \\
$\mathrm{PVR} / \mathrm{VV}$ & $0.51 \pm 0.6$ & $0.33 \pm 0.3$ & 0.11 \\
Qmax $(\mathrm{mL} / \mathrm{sec})$ & $8.3 \pm 3.3$ & $10.3 \pm 3.4$ & 0.016 \\
IPSS & $21.3 \pm 7.7$ & $20.5 \pm 10.4$ & 0.83 \\
QOL & $4.4 \pm 0.9$ & $4.1 \pm 1.1$ & 0.37 \\
\hline
\end{tabular}

Values are presented as mean \pm standard error.

$\mathrm{BPH}$, benign prostatic hyperplasia; VV, voiding volume; PVR, postvoid residual urine; Qmax, maximal flow rate; IPSS, International Prostate Symptom Score; QOL, quality of life.

Table 1. Characteristics of the patients in each group

\begin{tabular}{|c|c|c|c|c|c|c|}
\hline \multirow{2}{*}{ Parameter } & \multirow{2}{*}{ Total patient } & \multirow{2}{*}{$\begin{array}{c}\text { Group 1 } \\
(\mathrm{n}=43,13.9 \%)\end{array}$} & \multirow{2}{*}{$\begin{array}{c}\text { Group 2 } \\
(\mathrm{n}=266,86.1 \%)\end{array}$} & \multirow{2}{*}{$\begin{array}{c}\text { Univariate } \\
\text { P-value }\end{array}$} & \multicolumn{2}{|c|}{ Multivariate } \\
\hline & & & & & $\mathrm{P}$-value & Odds ratio \\
\hline Age (yr) & $67.5 \pm 7.8$ & $70.7 \pm 6.0$ & $66.9 \pm 7.9$ & $<0.01$ & 0.21 & 1.03 \\
\hline $\mathrm{VV}(\mathrm{mL})$ & $206 \pm 123$ & $194 \pm 123$ & $207 \pm 124$ & 0.51 & 0.83 & 1.0 \\
\hline $\operatorname{PVR}(\mathrm{mL})$ & $49.1 \pm 63.1$ & $76.7 \pm 70.6$ & $41.1 \pm 60.5$ & $<0.01$ & 0.04 & 1.005 \\
\hline $\mathrm{PVR} / \mathrm{VV}$ & $0.3 \pm 0.8$ & $0.6 \pm 0.7$ & $0.4 \pm 1.3$ & 0.19 & 0.44 & 0.78 \\
\hline Qmax (mL/sec) & $9.1 \pm 4.2$ & $8.1 \pm 3.1$ & $9.3 \pm 4.4$ & 0.03 & 0.61 & 0.97 \\
\hline IPSS & $18.2 \pm 7.2$ & $20.9 \pm 7.6$ & $17.8 \pm 7.1$ & 0.015 & 0.03 & 1.07 \\
\hline QOL & $3.9 \pm 1.2$ & $4.2 \pm 1.0$ & $3.9 \pm 1.2$ & 0.053 & 0.45 & 1.15 \\
\hline Prostate volume (g) & $29.5 \pm 18.2$ & $44.9 \pm 17.4$ & $27.0 \pm 17.1$ & $<0.01$ & $<0.001$ & 1.05 \\
\hline PSA (ng/dL) & $1.3 \pm 0.9$ & $1.7 \pm 0.9$ & $1.3 \pm 0.9$ & $<0.01$ & 0.73 & 1.08 \\
\hline Creatinine $(\mathrm{ng} / \mathrm{mL})$ & $1.1 \pm 0.2$ & $1.7 \pm 0.2$ & $1.1 \pm 0.2$ & 0.85 & 0.83 & 1.27 \\
\hline
\end{tabular}

Values are presented as mean \pm standard error.

VV, voiding volume; PVR, post-void residual urine; Qmax, maximal flow rate; IPSS, International Prostate Symptom Score; QOL, quality of life; PSA, prostate-specific antigen. 
1 with $90 \%$ specificity was $122 \mathrm{~mL}$, but the sensitivity at this point was only $21 \%$. The $72 \%$ sensitivity cutoff using PVR was $28.5 \mathrm{~mL}$, but the specificity at this point was limited as $67.3 \%$. Due to these limitations in identification of an acceptable cutoff with sufficient sensitivity and specificity at the same time, the whole patient group was redivided by arbitrarily selected cutoffs of $50 \mathrm{~mL}, 100 \mathrm{~mL}$, and $150 \mathrm{~mL}$. In this analysis, 51.2\% (22/43) of Group 1 had PVR over $50 \mathrm{~mL}, 25.6 \%$ (11/43) had PVR over $100 \mathrm{~mL}$, and 13.9\% (6/43) had PVR over $150 \mathrm{~mL}(\mathrm{P}<0.001$, 0.027 , and 0.13 , respectively, by chi-square test) (Table 3 ). The relative risk of a BPH-related clinical event using each PVR cutoff was measured as 3.93 (95\% confidence interval [CI], 2.02 to 7.65), 2.61 (95\% CI, 1.19 to 5.569), and 2.11 (95\% CI, 0.79 to $5.62)$, respectively. However, the majority of patients at each

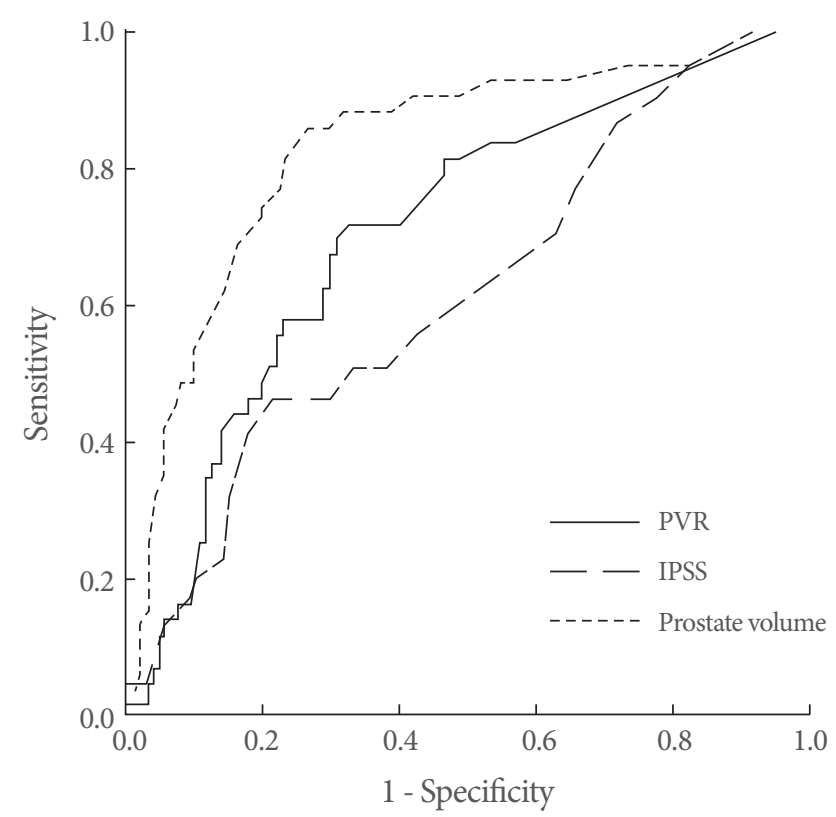

Fig. 1. Receiver-operating characteristic curve analysis of post voiding residual volume (PVR), International Prostate Symptom Score (IPSS), and prostate volume in patients with or without prostate surgery, acute urinary retention, urinary tract infection.
PVR cutoff were in Group 2: 71.8\% (56/78) with PVR over 50 $\mathrm{mL}, 73.8 \%$ (31/42) with PVR over $100 \mathrm{~mL}$, and 76\% (19/25) with PVR over $150 \mathrm{~mL}$.

\section{DISCUSSION}

The evaluation of patients with $\mathrm{BPH}$ requires a thorough understanding of the physiology of voiding and of the potential pathophysiologic changes. Despite the controversy over the use of PVR in BPH patients as a diagnostic and prognostic test, PVR continues to be used mainly because of its procedural simplicity, and has been thought to have a prominent role in identifying those with obstructions. However, the pathophysiology of PVR is not generally well understood, and interactions with BOO and detrusor contractility are complex. Although a lack of PVR clearly indicates that the patient can efficiently empty his bladder, it does not exclude the presence of BOO [10]. In the case of increased PVR, although it may result from BOO [11], differentiation of other causes, including deficient bladder contractility, as the primary cause of symptoms is impossible without a pressure-flow study. Moreover, a large PVR may reflect detrusor underactivity rather than BOO [12].

The aim of the present study was to evaluate the clinical implications of PVR as a prognostic factor for BPH progression. First, we measured baseline variables for all enrolled patients who visited our institution for LUTS and investigated the correlation of PVR with other variables. The data showed that PVR was correlated with Qmax, IPSS, prostate volume, and serum PSA. Similar to our results, PVR has been reported to have a statistically significant association with prostate volume [3], severity of symptoms, maximal flow rate [13], and age [14]. Kolman et al. [3] investigated PVR and prostate volume in 477 randomly selected community-dwelling white men and reported a significant correlation of PVR and prostate volume $(\mathrm{P}<0.001)$. The odds of PVR greater than $50 \mathrm{~mL}$ was 2.5 times greater for men with a prostate volume of $30 \mathrm{~mL}$ than for those with small-

Table 3. Division of each patient group dependent on each arbitrarily selected PVR cutoffs

\begin{tabular}{|c|c|c|c|c|c|c|}
\hline & \multicolumn{6}{|c|}{ PVR (mL) cutoff } \\
\hline & $\geq 50$ & $<50$ & $\geq 100$ & $<100$ & $\geq 150$ & $<150$ \\
\hline Group $1(n=43)$ & $22(51.2)$ & $21(48.8)$ & $11(25.6)$ & $32(74.4)$ & $6(13.9)$ & $37(86.1)$ \\
\hline Group $2(n=266)$ & $56(21.0)$ & $210(79.0)$ & $31(11.6)$ & $235(88.4)$ & $19(7.1)$ & $247(92.9)$ \\
\hline
\end{tabular}

Values are presented as number (\%).

PVR, post-void residual urine. 
er prostates. In an analysis of 198 patients after treatment of $\mathrm{BPH}$, Barry et al. [13]. demonstrated that reduction of the symptoms score was significantly correlated with improvement of uroflowmetry, including PVR. However, others have found no correlation between PVR and LUTS [15] and prostate volume [16]. Also, despite a correlation with prostate volume, Kolman et al. [3] found no significant relationship between PVR and Qmax, age, or symptom score. In our report, the relationships of PVR with other variables were very weak, with a coefficient of correlation ranging from 0.1 to 0.3 . These weak correlations are consistent with the findings of Kranse and van Mastrigt [17], who analyzed the relationship of PVR and the International Continence Society nomogram for the definition of BOO. In their report, areas under the ROC curve were estimated as 0.63 and 0.64 , respectively.

The poor baseline correlations shown in the above series indicate the unreliability of PVR as a single dependent parameter indicating BOO. Actually, correct interpretation of PVR measurement takes into consideration at least 2 aspects: the great variability in the male population and the significant number of asymptomatic patients with increased residual volume [18]. Physiologic measures always exhibit some degree of intra-individual variation, and Birch et al. [19] reported that of 30 men, $66 \%$ had significant variations in PVR when three scans were performed on the same day. In an attempt to decrease intra-individual variation, Memon and Ather [20] suggested the concept of residual fraction, reporting a more accurate and reproducible index of bladder emptying. The efficacy of this novel concept in the identification of $\mathrm{BOO}$ patients has been supported by other authors [21]. However, in this series, it showed no predictability in the identification of patients with clinical BPH progression. Considering these limitations, Kou [22] suggested the concept of the prostate score, which provided better sensitivity for patients with BOO, based on a combination of variables from uroflowmetry, including PVR and prostate volume measurement by TRUS.

In this study with a minimum 3-year follow up, we evaluated $\mathrm{PVR}$ as an indicator of BPH-related clinical events in members of the Korean population. Although most patients (86.1\%) were maintained with medication, patients with BPH-related clinical events had a significantly larger prostate volume, aggravated symptoms, and increased PVR at time of initial evaluation. In patients with a baseline PVR over $50 \mathrm{~mL}$, the relative risk of $\mathrm{BPH}$-related clinical events was 3.9 times greater than for those with a smaller PVR, and it was 2.6 times with a baseline PVR over $100 \mathrm{~mL}$. Particularly for PVR, these observations had already been previously reported in Western community-based longitudinal studies and placebo-controlled studies. In an Olmsted County study, which included 477 randomly selected men, men with a baseline PVR of $<50 \mathrm{~mL}$ were also three times as likely to develop subsequent AUR requiring catheterization during follow-up [3]. In the Medical Therapy of Prostatic Symptoms study, which included 3,047 men with moderate to severe LUTS, higher baseline PVR was associated with a slightly greater risk of developing BPH-related clinical progression [23]. In a recent Western longitudinal population-based study with a follow-up period of 6.5 years for 1,688 men, multivariate analysis showed that PVR was one of the independent determinants with a significant hazard radio in prediction of an increase of IPSS greater than 7 [5]. In this study, however, the relative risk of a $\mathrm{BPH}-$ related clinical event is high with more than the each PVR cutoff, but the dose-response relation is not shown. The reason seems to be that as PVR cutoff gets bigger the number of subject patients that have more than the cutoff gets smaller. This is a problem due to the fact that the number of patients of Group 1 is primarily too small.

One of the peculiarities of our data is the observation that baseline PVR in patients who underwent BPH-related surgery had not changed at the time of surgery, despite a mean interval of 13.1 months after the initial visit. As presented in Table 2, changes in data occurred only in Qmax, which increased after medication therapy. The unchanged IPSS in these data indicates that the unsatisfactory improvement of symptoms was the main reason for this surgical conversion. All patients enrolled had visited the clinic for evaluation of LUTS symptoms; thus, although Qmax increased after medication during the follow-up, nonresponders in terms of symptom score or PVR may be at risk of surgical intervention. However, due to the inherent limitations of retrospectively collected data, direct conclusions on this issue cannot be extracted. Finding answers to the above questions will require further investigation via comparative analysis.

Although the predictability of PVR for BPH progression was observed in this series, the very low odds ratio reflects the complexity of clinical interpretation of increased PVR. Actually, although no significant increase in baseline PVR in the BPH progress group and no change in PVR from baseline at the time of surgery were observed, the outcome of our attempt to determine clinically helpful cutoffs from these data was not satisfactory. Although the statistical tools proposed $28.5 \mathrm{~mL}$ as a cutoff level 
with $72 \%$ sensitivity and $67 \%$ specificity, this is not a clinically acceptable level, considering the lack of reproducibility of these tests [24]. Furthermore, the discrepancy between the actual amount of urine and PVR measured by ultrasonography has been reported to increase in the low-volume range of PVR [25]. In addition, although statistical significance was observed when the traditionally considered PVR cutoffs of $50 \mathrm{~mL}$ and $100 \mathrm{~mL}$ were applied to identify patients with $\mathrm{BPH}$ clinical progression [3], it is noteworthy that most of the patients at each cutoff level were maintained with medication therapy without the occurrence of BPH-related clinical events.

We still recognize several limitations in this series. As previously mentioned, the retrospective study design not only limited the collection of variables but also hindered consistent enrollment of patients. Decisions on BPH-related surgery were made on the basis of the preference of each physician and patient rather than strict guidelines. Pressure-flow study, which can definitely discriminate patients with $\mathrm{BOO}$, was not evaluated. As with other community-based studies, uroflowmetry and PVR measurement were conducted only one time per event. Changes in PVR in patients without BPH-related clinical events during follow-up were not evaluated serially. Because all patients enrolled had LUTS, this outcome of PVR cannot be generalized to patients without voiding symptoms. Our data did not suggest a reasonable cutoff value for PVR to discriminate patients. In addition, this was a single-center study based on patients who visited a third-degree medical center.

In conclusion, our data with a minimum of 3 years of followup indicate that increased PVR at the baseline evaluation is a significant indicator of BPH-related clinical events along with increased IPSS or prostate volume in symptomatic members of the Korean population. In patients who required BPH-related surgery, although maximal flow rate improved after medical treatment, the symptom score and amount of PVR did not change during follow-up. However, our inability to provide a clinically acceptable cutoff implies the unreliability of PVR as a single dependent parameter indicating BOO. Further investigations with proper designs to compensate for these limitations are required the take advantage of the obvious usefulness of the noninvasive variable of PVR in patients with $\mathrm{BPH}$.

\section{CONFLICT OF INTEREST}

No potential conflict of interest relevant to this article was reported.

\section{REFERENCES}

1. Emberton M. Definition of at-risk patients: dynamic variables. BJU Int 2006;97 Suppl 2:12-5.

2. Xia SJ, Xu XX, Teng JB, Xu CX, Tang XD. Characteristic pattern of human prostatic growth with age. Asian J Androl 2002;4:269-71.

3. Kolman C, Girman CJ, Jacobsen SJ, Lieber MM. Distribution of post-void residual urine volume in randomly selected men. J Urol 1999;161:122-7.

4. Kok ET, Schouten BW, Bohnen AM, Groeneveld FP, Thomas S, Bosch JL. Risk factors for lower urinary tract symptoms suggestive of benign prostatic hyperplasia in a community based population of healthy aging men: the Krimpen Study. J Urol 2009;181:710-6.

5. Crawford ED, Wilson SS, McConnell JD, Slawin KM, Lieber MC, Smith JA, et al. Baseline factors as predictors of clinical progression of benign prostatic hyperplasia in men treated with placebo. J Urol 2006;175:1422-6.

6. Kristal AR, Arnold KB, Schenk JM, Neuhouser ML, Weiss N, Goodman P, et al. Race/ethnicity, obesity, health related behaviors and the risk of symptomatic benign prostatic hyperplasia: results from the prostate cancer prevention trial. J Urol 2007;177:1395-400.

7. Jin B, Turner L, Zhou Z, Zhou EL, Handelsman DJ. Ethnicity and migration as determinants of human prostate size. J Clin Endocrinol Metab 1999;84:3613-9.

8. Oesterling JE, Kumamoto Y, Tsukamoto T, Girman CJ, Guess HA, Masumori N, et al. Serum prostate-specific antigen in a community-based population of healthy Japanese men: lower values than for similarly aged white men. Br J Urol 1995;75:347-53.

9. Simforoosh N, Dadkhah F, Hosseini SY, Asgari MA, Nasseri A, Safarinejad MR. Accuracy of residual urine measurement in men: comparison between real-time ultrasonography and catheterization. J Urol 1997;158:59-61.

10. Bates P, Bradley WE, Glen E, Griffiths D, Melchior H, Rowan D, et al. The standardization of terminology of lower urinary tract function. J Urol 1979;121:551-4.

11. Ball AJ, Feneley RC, Abrams PH. The natural history of untreated “prostatism". Br J Urol 1981;53:613-6.

12. Van Mastrigt R, Rollema HJ. The prognostic value of bladder contractility in transurethral resection of the prostate. J Urol 1992;148: 1856-60.

13. Barry MJ, Cockett AT, Holtgrewe HL, McConnell JD, Sihelnik SA, Winfield HN. Relationship of symptoms of prostatism to commonly used physiological and anatomical measures of the severity of benign prostatic hyperplasia. J Urol 1993;150(2 Pt 1):351-8.

14. Ruud Bosch JL. Postvoid residual urine in the evaluation of men 
with benign prostatic hyperplasia. World J Urol 1995;13:17-20.

15. Cetinel B, Turan T, Talat Z, Yalçin V, Alici B, Solok V. Update evaluation of benign prostatic hyperplasia: when should we offer prostatectomy? Br J Urol 1994;74:566-71.

16. Bruskewitz RC, Iversen P, Madsen PO. Value of postvoid residual urine determination in evaluation of prostatism. Urology 1982;20: $602-4$.

17. Kranse R, van Mastrigt R. Weak correlation between bladder outlet obstruction and probability to void to completion. Urology 2003;62: 667-71.

18. Bonde HV, Sejr T, Erdmann L, Meyhoff HH, Lendorf A, Rosenkilde $\mathrm{P}$, et al. Residual urine in 75-year-old men and woman: a normative population study. Scand J Urol Nephrol 1996;30:89-91.

19. Birch NC, Hurst G, Doyle PT. Serial residual volumes in men with prostatic hypertrophy. Br J Urol 1988;62:571-5.

20. Memon A, Ather MH. Use of residual fraction instead of residual volume in the evaluation of lower urinary tract symptoms. Tech
Urol 2000;6:26-8.

21. Ku JH, Cho SY, Oh SJ. Residual fraction as a parameter to predict bladder outlet obstruction in men with lower urinary tract symptoms. Int J Urol 2009;16:739-44.

22. Kuo HC. Clinical prostate score for diagnosis of bladder outlet obstruction by prostate measurements and uroflowmetry. Urology 1999;54:90-6.

23. Emberton M, Elhilali M, Matzkin H, Harving N, van Moorselaar J, Hartung R, et al. Symptom deterioration during treatment and history of AUR are the strongest predictors for AUR and BPH-related surgery in men with LUTS treated with alfuzosin $10 \mathrm{mg}$ once daily. Urology 2005;66:316-22.

24. Reilly NJ. The release of the clinical practice guideline: benign prostatic hyperplasia: diagnosis and treatment. Urol Nurs 1994;14:37.

25. Roehrborn CG, Peters PC. Can transabdominal ultrasound estimation of postvoiding residual (PVR) replace catheterization? Urology 1988;31:445-9. 